letters strongly support the need for such research.

Our own primary hypothesis is that healthcare planners have used the easy access to the frontline doctor as an excuse for long waiting lists for complicated clinical trajectories. But we also raise the question whether we as GPs have found the correct balance between necessary investigations and the protection of patients against unnecessary investigations. This is an important, but yet not fully answered, research question that is also raised in the letter from Polak. ${ }^{2}$ We sincerely support the call for more research in the net effect of better access to diagnostic procedures.

Polak also points to the crucial question whether people may have an increasing perception of GPs as being rationing 'keepers' only. That this may have an effect on the way patients seek help is in accordance with new research. ${ }^{3}$ People may think that the GP is some kind of 'barrier' to medical care access and thus postpone attending the GP. Or people experience their nice and friendly GP as very busy and do not want to disturb them, exactly the point also made by Davies. ${ }^{4}$

One of the challenges in comparing differences between countries is the possible difference in how diagnoses are registered. Treasure ${ }^{5}$ has a very important methodological point in asking whether the 1 -year survival is higher in the nongatekeeper systems simply due to lead time bias. Lead time bias is definitely a possible explanation for the outcome of these types of comparative studies. The question is, however, whether such lead time bias can explain differences between countries of $5-10 \%$ in relative survival. A recent simulation study ${ }^{6}$ found that the difference in registration of a breast cancer diagnosis should be unlikely large if it should explain the differences between UK and Sweden. However, the effect of lead time bias in comparisons needs much more rigorous research.

We strongly agree with Davies that our data should be replicated using newer data and also data on other serious diseases. New research should also address if different remuneration systems may have impact on the quality of gatekeeping.

We were happy to see three academically well argued responses to our paper. A fourth response by Manassiev lposted on the BJGP Discussion Forum) seems to be very little in favour of discussing whether there could be side effects of gatekeeping. In many ways the response speaks for itself. In a proper way we point out that our study is an ecologic study.
The use of quintiles in the paper by Møller et al does not change anything as we used this in our calculations. We do not think that use of the 1- and 5-year relative survival of lip cancer would improve the paper as suggested by Manassiev. Manassiev may have different memories about gatekeeping in some countries, but we prefer research published in the literature. We have written our arguments for the use of 1-year survival and we kindly ask our readers to check them and compare with Manassiev's not quite academic approach. It is not correct that the majority of sufferers of the top four cancers (lung, breast, prostate, and colon) would survive 1 year probably whatever the health system'. We do not agree with Manassiev about the incompetence of editors and reviewers and we trust that many readers are able to read papers without having passed Manassiev's research school on the shape of the earth.

In conclusion, we must realise that general practice has several key roles. One important role is to be aware of new, rare, but serious diseases that, in a timely way, should be guided through the healthcare system without delay that may influence prognosis. We need much more research on the impact of different organisational models on this key role. $^{8}$

\section{Peter Vedsted,}

Aarhus University, Research Unit for General Practice, Bartholins Allé 2, Aarhus, 8000, Denmark.

E-mail: p.vedstedlaalm.au.dk

Frede Olesen,

University of Aarhus, Research Unit for General Practice, Aarhus C, 8000, Denmark.

\section{REFERENCES}

1. Vedsted $P$, Olesen F. Are the serious problems in cancer survival partly rooted in gatekeeper principles? An ecologic study. Br J Gen Pract 2011; DOI: 10.3399/bjgp11X588484.

2. Polak L. Are the serious problems in cancer survival partly rooted in gatekeeper principles? [Letter] $\mathrm{Br} J$ Gen Pract 2011; 61(592): 661.

3. Andersen RS, Vedsted P, Olesen F, et al. Does the organizational structure of health care systems influence care-seeking decisions? A qualitative analysis of Danish cancer patients' reflections on care-seeking. Scand J Prim Health Care 2011; 29(3): 144-149

4. Davies P. Are the serious problems in cancer survival partly rooted in gatekeeper principles? [Letter] $\mathrm{Br} \mathrm{J}$ Gen Pract 2011; 61(592): 661.

5. Treasure W. Are the serious problems in cancer survival partly rooted in gatekeeper principles? [Letter] Br J Gen Pract 2011; 61(592): 661.
6. Woods LM, Coleman MP, Lawrence G, et al. Evidence against the proposition that 'UK cancer survival statistics are misleading': simulation study with National Cancer Registry data. BMJ 2011; 342: d3399.

7. Møller $\mathrm{H}$, Linklater KM, Robinson D. A visual summary of the EUROCARE-4 results: a UK perspective. Br J Cancer 2009; 101 (Suppl 2): S110-114.

8. Olesen F. Putting research into primary care practice. BMJ 2011; 343: d3922

DOI: 10.3399/bjgp11X606537

\section{Extent of cotton-bud use in ears}

The use of cotton buds inside ears has widely been condemned worldwide by otolaryngologists. This is due to well documented complications including trauma, impacted ear wax, infection, and retention of the cotton bud. ${ }^{1}$ The most common mode of accidental penetrating ear injury in children is cotton-bud induced. ${ }^{2}$ Trends in cotton-bud usage have been studied previously, but have only focused on ear, nose, and throat (ENT) patients. ${ }^{3}$ In recent times, manufacturers have heeded the advice of the otolaryngologist and have consequently relayed warnings to the public. We conducted a survey to investigate the extent of 'Q-tip' cotton bud public use in ears and the awareness of associated complications.

Between January and August 2009, confidential questionnaires were given to patients at three primary care centres in the south east of England. The response rate was $80 \%$ (239/300). Ages ranged from 17 to 87 years, with a mean of 41.1 years. There were 144 (60\%) female and 95 (40\%) male responders. An alarming $68 \%$ admitted using cotton buds in their ears, with $76 \%$ of users using them at least weekly, if not more frequently. The primary reason (96\%) given for using cotton buds was to remove earwax.

It is evident that there is a public perception that the ear requires regular cleaning. However, our knowledge dictates that earwax is produced in the outer part of the canal and migrates out with the epithelium towards the pinna. Other reasons included relieving an itch and drying the ear. Our survey showed that cotton-bud users were aware of $52 \%$ of the potential complications, whereas non cotton-bud users were aware of $59 \%$ of potential complications. There was no significant association between awareness of complications and cotton-bud use $l \chi^{2}=2.23$, $\mathrm{df}=3, P=0.53)$.

Despite manufacturers' warnings, use of 
cotton buds inside ears seems to be common. Our survey was carried out in the south east of England, as a result there may be a population bias. Further research into both adult and paediatric populations country-wide is warranted. One previous study did attempt to evaluate cotton-bud use and awareness of complications. ${ }^{3}$ As responders were ENT-clinic attendees, the study was limited by a biased sample. However, similar levels of cotton-bud use and awareness of complications were found.

Awareness of cotton-bud related complications is an important public health issue. We recommend that public awareness of cotton-bud related complications and the notion of the 'self-cleaning' ear needs to be raised. A small proportion of patients do suffer from regular ear wax impaction. Safer methods of aural toileting that include syringing and microsuction may also need promoting. With the above information, the public can make an informed choice of whether or not to use cotton buds. One method of promoting awareness may be to distribute leaflets in primary care centres. This may reduce cotton-bud related complications in the community.

\section{Sidhartha Nagala,}

ENT Research Fellow, Addenbrookes

Hospital, University of Cambridge.

E-mail: sn391@acam.ac.uk

Pranay Singh,

ENT Specialist Registrar, Ashford Hospital, Kent.

Philippa Tostevin,

ENT Consultant, St George's Hospital Medical School, London.

\section{Acknowledgements}

We would like to thank patients and staff in the three primary care centres in which the survey was carried out.

\section{REFERENCES}

1. Raman R. Should cotton buds be banned? Trop Doct 1997; 27(4): 250.

2. Steele BD, Brennan PO. A prospective survey of patients with presumed accidental ear injury presenting to a paediatric accident and emergency department. Emerg Med J 2002: 19(3): 226-228.

3. Hobson JC, Lavy JA. Use and abuse of cotton buds. J R Soc Med 2005; 98(8): 360-361.

DOI: 10.3399/bjgp11X606546

\section{Inequalities in general practice website provision}

Beasley et al' in their editorial describe how the implementation of electronic health records (EHR) is a transformative change. Some practices have made that transformation. For example, Amir Hannan and colleagues at Harold Shipman's previous practice at Haughton Thornton Medical Centre (http://www.htmc.co.uk), not only use EHR but offer their patients many e-health facilities, such as access to their own records, booking of appointments, repeat prescriptions, advice on preparing for the consultation, as well as links to numerous resources both locally and nationally. GP system suppliers provide patient directed web-based functions, such as repeat prescribing and patient access to records that, technically, just need to be 'turned on'. However, practices using these functions are in the minority. A third of practices have yet to offer their patients use of a practice website.

We examined the geographical variation in practice website provision in August 2011. We used data from NHS Choices on 8399 practices in England. We chose a purposive sample of 1026 practices in 14 postcode areas across England that were likely to include areas with high, medium, and low provision of GP websites. We used practice name and address to search Google ${ }^{\text {TM }}$ for a practice website. The accuracy of searching was checked by an observer variation study on a sub-sample of 50 . There was agreement on 46/50 (Kappa $=0.81)$.

Two-thirds (676/1026) of practices had a website that could be found on Google. This varied from $94 \%$ (all but one practice) in Harrogate to 35\% in Southend (Table 1). We did not assess the functionality of the websites but anecdotally know that many websites were just 'electronic nameplates' rather than functional sites for patient use.

Discussion about digital health inequalities tends to focus on the access that patients may have to the internet or in their ability to use it, ${ }^{2-}$ ${ }^{4}$ but there are clearly inequalities in the provision of opportunity to use e-health. The online facilities that are offered at some practices should be offered to all and should be considered a mark of a quality practice. Primary care trusts or GP cooperatives or whoever is now responsible in areas such as Southend, Wakefield, Blackburn, and Fylde should examine why their patients should have such poor opportunity for e-health. We need to explore with all stakeholders in
Table 1. Website provision

\begin{tabular}{lccc} 
& $\begin{array}{c}\text { Postcode } \\
\text { area }\end{array}$ & $\begin{array}{c}\text { No website } \\
\boldsymbol{n}(\%)\end{array}$ & $\begin{array}{c}\text { Website } \\
\boldsymbol{n}(\%)\end{array}$ \\
\hline Harrogate & HG & $1(6)$ & $16(94)$ \\
\hline Taunton & TA & $5(10)$ & $43(90)$ \\
\hline Southampton & SO & $10(13)$ & $68(87)$ \\
\hline Bristol & BS & $19(16)$ & $100(84)$ \\
\hline Halifax & HX & $5(22)$ & $18(78)$ \\
\hline Plymouth & PL & $18(22)$ & $64(78)$ \\
\hline Sunderland & SR & $13(26)$ & $38(74)$ \\
\hline St Albans & $\mathrm{AL}$ & $6(26)$ & $17(74)$ \\
\hline London SW & SW & $48(32)$ & $101(68)$ \\
\hline Leeds & LS & $47(39)$ & $73(61)$ \\
\hline Fylde & FY & $23(50)$ & $23(50)$ \\
\hline Blackburn & BB & $48(51)$ & $46(49)$ \\
\hline Wakefield & WF & $39(55)$ & $32(45)$ \\
\hline Southend & SS & $68(65)$ & $37(35)$ \\
\hline Total & & $350(34)$ & $\mathbf{6 7 6 ( 6 6 )}$ \\
\hline
\end{tabular}

primary care - GPs, their staff, but also patients and carers - the opportunities and barriers to implementation of e-health methods.

Ray Jones,

Professor of Health Informatics, Faculty of Health, Education, and Society, Plymouth University, 3 Portland Villas, Drake Circus, Plymouth, PL4 8AA.

E-mail: R.Jones-5laplymouth.ac.uk

Lesley Goldsmith,

Research Fellow, Faculty of Health, Education and Society, Plymouth University, Plymouth.

\section{Anita O'Connor,}

Research Assistant, Faculty of Health, Education and Society, Plymouth University, Plymouth.

\section{Conflict of interest}

We are all patients in primary care.

\section{REFERENCES}

1. Beasley JW, Holden RJ, Sullivan F. Electronic health records: research into design and implementation. Br J Gen Pract 2011; 61(591): 604-605.

2. The Royal Society. Digital healthcare: the impact of information and communication technologies on health and healthcare. London: The Royal Society, 2006.

3. Del Hoyo-Barbolla E, Kukafka R, Arredondo MT, Ortega M. A new perspective in the promotion of ehealth. In: Hasman A, Haux R, van der Lei J, (eds.). Ubiquity: technologies for better health in aging societies. The Netherlands: IOS Press, 2006: 404-412.

4. Viswanath K, Kreuter MW. Health disparities, communication inequalities, and eHealth. Am J Prev Med 2007; 32(5 Suppl): S131-S133.

DOI: 10.3399/bjgp11X606555 